\title{
ACCELERATION THROUGH X-TYPE COALESCENCE IN A SMALL ENERGETIC FLARE
}

\author{
Jun-Ichi Sakai ${ }^{(1.2)}$ and Cornelis de Jager ${ }^{(2)}$ \\ (1) Dept. of Applied Mathematics and Physics; \\ Faculty of Enginnering, Toyama University, Toyama 930, JAPAN \\ (2) SRON Laboratory for Space Research, and Astronomical \\ Institute, Beneluxlaan 21, 3527 HS Utrecht, The NETHERLANDS
}

\begin{abstract}
We studied the acceleration conditions in the small but fairly energetic flare of May 21, 1984 at $13: 26 \mathrm{UT}$. The most pronounced aspect of this flare was a series of 13 microwave / x-ray spikes, each lasting for about $0.1 \mathrm{~s}$. A previous study has shown that each of these was due to a series of successive sudden formations of small plasma knots of high energy particles. Each of these knots lost its energy in about $50 \mathrm{~ms}$. In the present study we show that these knots can originate by the process of $\mathrm{x}$-type (3-D) flux tube coalescence. The predicted rise time (30 to 50 $\mathrm{ms}$ ) and energy are in good agreement with the observationally derived parameters.
\end{abstract}

\section{Introduction}

On May 21, 1984 at 13 : 25 UT a solar flare was observed in microwavec and hard x-rays, which was in many respects remarkable (Kaufmann et al., 1985). An interesting aspect of the flare was its short duration (only one minute in $\mathrm{x}$-and radio-waves) but most remarkable was one of its flare burst which lasted for about three seconds. At millimeter waves $(90 \mathrm{GHz})$ this short burst was the strongest burst of the flare. In x-rays it emitted at higher energies than the other bursts of the flare complex, with a noticiable radiation component above $100 \mathrm{keV}$. Its most interesting aspect was that at $90 \mathrm{GHz}$ the burst consisted of about 13 short-lived spikes, each with a lifetime of about $0.1 \mathrm{~s}$. The spikes were about symmetrical in their time profiles, having rise-and decay-times of about $60 \mathrm{~ms}$.

Several explanations have been proposed for this high-eneray component of the flare (Kaufmann et al., 1985,1986; De Jager et al., 1987) . In the latter paper it was shown that each of the $100 \mathrm{~ms}$ spikes could be explained by the following emission model: The individual spikes, both in microwaves and in hard $\mathrm{x}$-rays were produced by the same electron population: the $\mathrm{x}$-rays by their bremsstrahlung, and the microwaves by their gvrosynchrotron emission. The emission sources were small, with a characteristic size of about $350 \mathrm{~km}$. They had an electron density of about $10^{11} \mathrm{~cm}^{-3}$, a 'temperasure' of $5 \times 10^{8} \mathrm{~K}$ and a magnetic field of about 1400-2000 G. Energy 
injection should have occurred in less than about $50 \mathrm{~ms}$ (as judged from the rise times of the spikes). It could be shown that plasma knots with the character described above should loose their energy by saturated convective flux (collisionless conduction) in about $30 \mathrm{~ms}$, a time which is good agreement with the observed decay times of the spikes $(60 \mathrm{~ms})$. The fact that 13 spikes were observed in a rapid succession then leads automatically to the conclusion that this was due to the successive formation of 13 plasma knots as described above. These successive energizations could occur by successive interaction between many small flux tubes ('flux threads') which are assumed to occur in the flaring region and which interact, a process that way occur by external forces acting on and deforming the system of flux threads. Since the thermal energy of the flare knots was about $10^{26} \mathrm{erg}$, this must be the amount of released in each of the flux-thread interactions.

Hence, the emission mechanism of the bursts can be understood. The problem remains how the bursts originated. It will be shown in this Letter that this aspect can be explained by the mechnism of $\mathrm{x}$-type (3-D imensional) flux tube coalescense, a mechanism presently being studied by one of the authors (Sakai and de Jager, 1990.). We note here that the mechanism of flux-tube coalescence has been described and studied earlier (see review by Sakai and Ohsawa, 1987), but so far only for the one-and two-dimensional cases. In the present (x-type) case we are dealing with two crossed flux-tubes, interacting in one point; a fundamentally new process as compared to the 1-D and 2-D cases.

\section{Energetics; conditions suitable for coalescence}

A spherical region with a diameter of $350 \mathrm{~km}$ and a magnetic field $\mathrm{B}$ of 1400 to $2000 \mathrm{G}$ would contain a magnetic energy $\mathrm{B}^{2} \mathrm{~V} / 8 \pi$, being to being 2 to $4 \times 10^{27} \mathrm{erg}$. The thermal energy content of that volume, assuming an electron density. $\mathrm{n}_{e}=10^{11}$ and temperature $\mathrm{T}=5 \times 10^{8} \mathrm{~K}$, is $1.5 \times 10^{26}$ erg. Hence the magnetic energy of the region is sufficient to provide the energy of the flare spikes.

In order to know if the conditions in the flaring area are suitable for explosive coalescence one has to know the value of $\beta=\left(c_{\mathrm{s}} / \mathrm{v}_{\mathrm{A}}\right)^{2}$. Here, $\mathrm{c}_{\mathrm{s}}$ is the sound velocity and $\mathrm{v}_{\mathrm{A}}$ the Alfven velocity. The value of $\mathrm{v}_{\mathrm{A}}$ has to be calculated using the magnetic field produced by the loop current (and not the ambient field.). With again $\mathrm{n}_{\mathrm{e}}=10^{11} \mathrm{~cm}^{-3}$ and taking $\mathrm{B}=1500 \mathrm{G}$ and $\mathrm{T}=5 \times$ $10^{8} \mathrm{~K}$ we find $\mathrm{v}_{\mathrm{A}}=2000 \mathrm{~km} \mathrm{~s}^{-1}$ and $\mathrm{c}_{\mathrm{S}}=20000 \mathrm{~km} \mathrm{~s}^{-1}$. Hence the value of $\beta$ after coalescence is $\beta=0.01$, with some uncertainty: $\mathrm{v}_{\mathrm{A}}$ is proportional to $\mathrm{B}^{-2}$ and to $\mathrm{T}$. It seems safe to assume $\beta<<$ 1 (after the coalescence). This is a condition that would be suitable for explosive coalescence if it would apply to the pre-coalescence situation. For large values of $\beta$, close to unity, our numerical calculations show that the coalescence would not be explosive in character.

\section{X-type coalescence applied to the 21 May 1984 bursts}

The $\beta$ value given above ( 0.01$)$ is the value that occurs after the explosion, but for a study 
of the coalescence process we are interested in the value before the start of the coalescence. These $\beta$ values may be larger than 0 . 01.: Detailed numerical calculations are so for only available for the case $\beta=0.5$. To start with, we will work with this value. Below we will find it to be a good approximation. The calculations show that after coalescence the particle density has increased by a factor 10 and the field strength $B$ (produced by the current loop) by a factor 100 . The fieldstrength $\mathrm{B}$ along the loop would increase by a factor 10 . That means that the precoalesence value. of $\mathrm{v}_{\mathrm{A}}$ should in that case have been about $300 \mathrm{~km} \mathrm{~s}^{-1}$. Assuming a coronal temperature of $10^{2} \mathrm{ev}$ before the coalescence, one would have $c_{\mathrm{s}}=100 \mathrm{~km} \mathrm{~s}^{-1}$. Hence the initial (pre-coalescence) value of $\beta$ would have been 0.1 , which is not too far from our tentative value $\beta=0.5$.

In such conditions the Alfven transit time over an area of $350 \mathrm{~km}$ would be about 1 second. Numerical calculations, given in Figures 1 and 2, show that the duration of the coalescent explosion is of the order of 0.03 to 0.05 Alfven transit times. Since the latter is about 15 , the explosion should have lasted for 30 to $50 \mathrm{~ms}$, as was indeed observed.

The numerical calculations also show that for $\beta=0.5$ the maximum explosion velocity is about $200 \mathrm{v}_{\mathrm{A}}$, which is about $60000 \mathrm{~km}$ $\mathrm{s}^{-1}$. This corresponds with an electron energy still below $100 \mathrm{keV}$; but if the initial $\beta$ value would have been 0.1 (the more likely case) then the explosion velocity would have been $\mathrm{c} / 3$, vilding electron energies above 100 $\mathrm{keV}$, as observed. We note that, as stated above, $\beta=0.1$ is indeed the most probable pre-coalescence value.
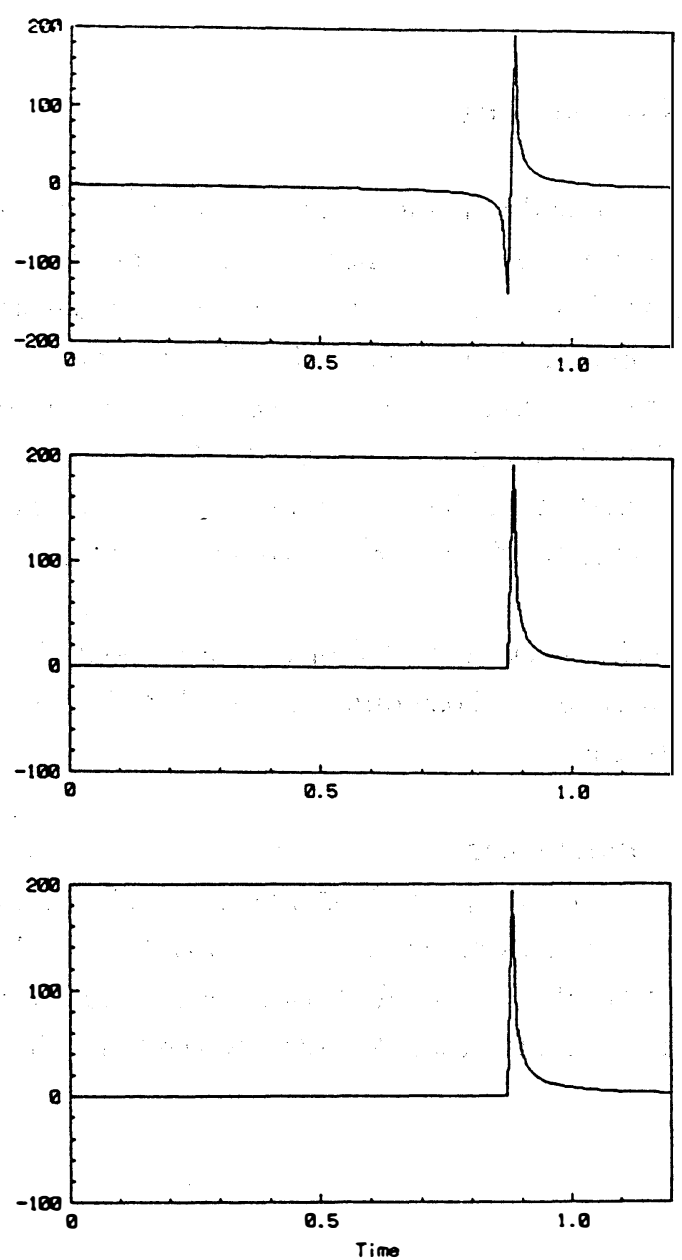

Fig. 1 Velocities in X-type (3-D) coalescence, calculated for the case $\beta=0.5$. The three diagrams show the velocities in the 3 cartesian coordinates. The upper diagram gives the velocity component in the direction of coalescence ; the two others in directions perpendicular to it. Horizontal axis: time in units of the Alfven transit time; Vertical axis: velocities in units of the Alfven velocity.

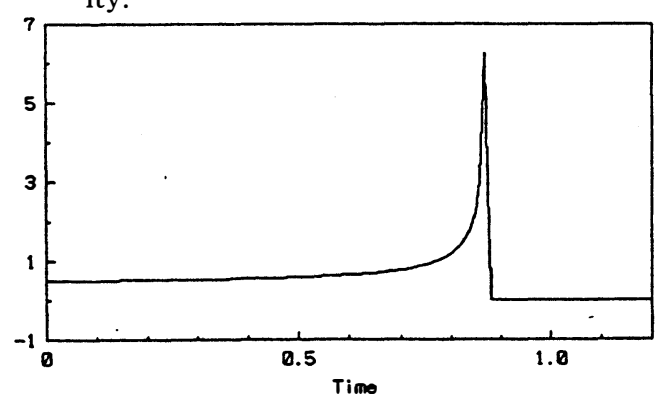

Fig. 2 Density in X-type coalescence. horizontal axis: time in units of the Alfven transit time ;vertical axis: density in arbitrary units. The initial density is 0.5 units. 


\section{Conclusions}

We found that the conditions in the area producing the remarkable flare bursts of 21 May 1984 were indeed such that the many flare spikes could have been due to explosive coalescence. The pre-flare conditions were an electron density of $10^{10} \mathrm{~cm}^{-3}$ and a magnetic field produced by the loop current of about $15 \mathrm{G}$. The magnetic field along the loop must have been $150 \mathrm{G}$. This is so because in the case $\beta=0.5$ coalescence causes the field along the loop to increase by a factor 10 while the magnetic field due to the loop current increases by a factor 100 . It seems furthermore likely that the interacting flux threads must have had diameters of the order of $350 \mathrm{~km}$, other wise the flare kernels would have been larger in size.

Acknowlegment: One of us (J.S.) wuold like to thank N.W.O. for a visiting fellowship. He ackowledges the hospitality of Prof. J.A.M. Bleeker his stay at the Laboratory for Space research in Utrecht.

\section{References}

De Jager, C., Kuijpers, J., Correia, E. and Kaufmann, P. : 1987, Solar phys., 110, 317.

Kaufmann, P., Correia, E., Costa, J.E.R., Vaz, A.M. and Dennis, B.R. : 1985, Nature, 313, 380.

Kaufman, P., Correia, E., Costa, J.E.R., and Zodi Vaz, A.M. : 1986, Astron. Astrophys. 157, 11. Sakai, J. and Ohsawa, Y.: 1987, Space Sci. Rev. 46, 113. 